\title{
Simultaneous Ipsilateral "Floating Hip" with Hip Dislocation and Femoral Neck and Shaft Fractures Associated with "Floating Knee" Injuries with Patella Fracture and Traumatic Sciatic Nerve Injury
}

\author{
Sasa S. Milenkovic ${ }^{a, b}$ Milan M. Mitkovic ${ }^{a, b}$ \\ a University of Nis, Faculty of Medicine, Nis, Serbia; ${ }^{b}$ Clinic for Orthopaedic surgery and \\ Traumatology, University Clinical Centre Nis, Nis, Serbia
}

Keywords

Injury · Floating hip · Floating knee

\begin{abstract}
Simultaneous ipsilateral "floating-hip" and "floating-knee" injuries are very rare and severe, and they occur in high-velocity road traffic accidents. A 55-year-old man presented with posterior wall fracture - dislocation of the acetabulum, complete fracture - dislocation of the femoral head, ipsilateral femoral shaft fracture, open patellar fracture, Gustilo type II, tibial fracture, and traumatic sciatic nerve injury/peroneal division. Given the fact that hip dislocation is an orthopedic emergency, we first did closed external tibial fixation, femoral head reduction, osteosynthesis of the acetabular fracture, and partial patellectomy. After 2 days, the patient underwent a second surgery; fixation of the neck and femoral shaft fractures was done, with a self-dynamic internal fixator. After 14 months from the injuries, radiographs show complete healing of all fractures, the patient walks independently without crutches, and the peroneal nerve is partially recovered. Despite the seriousness of the presented injuries, we did not have any complications, and 14 months after the injury, the femoral head is still viable, with no signs of femoral head osteonecrosis.
\end{abstract}




\section{Case Reports in Orthopedic Research}

\begin{tabular}{l|l}
\hline Case Rep Orthop Res 2021;4:255-260 \\
\hline DOI: 10.1159/000521029 & $\begin{array}{l}\text { @ 2021 The Author(s). Published by S. Karger AG, Basel } \\
\text { www.karger.com/cio }\end{array}$ \\
\hline Milenkovic and Mitkovic: Simultaneous Ipsilateral "Floating-Hip" and "Floating-Knee"
\end{tabular} Milenkovic
Injuries

\section{Introduction}

Simultaneous ipsilateral "floating-hip" and "floating-knee" injuries are very rare and severe, and they occur in high-velocity road traffic accidents. These injuries can be life-threatening, and many complications can occur during and after treatment. Simultaneous ipsilateral "floating-hip" and "floating-knee" injuries represent a great challenge and require extensive surgical experience, careful planning, and teamwork because there are no special protocols for the treatment of serious injuries such as these [1-4].

\section{Case Presentation}

A 55-year-old male was admitted due to simultaneous ipsilateral "floating hip" with hip dislocation, femoral neck and shaft fractures associated with "floating-knee" injuries with an open patella fracture, and traumatic sciatic/peroneal division injury. The injuries occurred in a high-velocity road traffic accident. Initially, the patient was hospitalized in a general hospital $200 \mathrm{~km}$ away, where radiographic diagnosis for his injuries (Fig. 1a-e) and initial fluid resuscitation were done. He was transported to our trauma center after a few hours. The injuries occurred on Friday, right before the upcoming weekend. On admission to the trauma center, he was hemodynamically unstable (TA $90 / 60 \mathrm{~mm} \mathrm{Hg}$, pulse $125 / \mathrm{min}$, erythrocytes 2.74 , hemoglobin, 78, hematocrit 0.24, Sp02 94\%, and serum lactate $2.5 \mathrm{mmol} / \mathrm{L}$ ). We started resuscitation with 2,000 mL of crystalloid, 3 units of blood transfusion, and $440 \mathrm{cc}$ of blood plasma. After aggressive resuscitation, the general condition was stabilized. A 3D CT of the acetabulum was done, and it showed a fracture of the posterior wall of the acetabulum with a fracture of the femoral neck and dislocation of the left hip (Fig. 1f, g). The patient also had an ipsilateral fracture of the femoral shaft, an open patella fracture (Gustilo-Anderson, grade II), and a tibial fracture. In the early morning hours of the next day, on Saturday, the patient was hemodynamically stable, but he underwent surgery after $72 \mathrm{~h}$, on the Monday morning. The serum lactate value was $1.9 \mathrm{mmol} / \mathrm{L} 72 \mathrm{~h}$ after injuries. Before the surgery, we found out that the patient had a sciatic nerve/peroneal division injury. After achieving hemodynamic stability in the patient, the surgeon should decide about the order of surgical treatment and the choice of implants for bone fixation of ipsilateral fractures of the femoral neck, femoral shaft, and tibia. We opted for staging surgery. First, we did the unilateral external fixation of the tibial fracture. Then, we placed the patient in a lateral decubitus, approached the left acetabulum, and found a completely extruded femoral head/neck separated from the capsule. The femoral head was found between the muscle fibers of the gluteus maximus muscle (Fig. 2a, b). We did the reduction of the femoral head (Fig. 2c), and intraoperatively, we identified a hematoma on the sciatic nerve (Fig. 2d). We performed reduction and osteosynthesis of the posterior wall of the acetabulum (Fig. 2e). At the end of this surgical procedure, the patient was returned to the supine position, and we performed a partial patellectomy due to the comminution of the distal part of the patella and reinsertion of the patellar ligament for the proximal part of the patella. Postoperatively, we placed the traction over the external fixator. On the third day after the first surgery (Fig. 3a), we performed the osteosynthesis of the femoral neck and shaft fractures. Under general anesthesia, the patient was placed in the supine position on an orthopedic table with extension and under fluoroscopic control, the femoral neck and femoral shaft fractures were fixed with a self-dynamizable internal fixator (Fig. 3b). Postoperative X-rays after external fixation of the tibial fracture are shown in Figure. 3c, d. At the end of the first week, we started with the patient verticalization. The patient was discharged from the hospital 18 days after admission. We started with progressive to full weight-bearing 2.5 months after the injury. Follow-up X-rays and clinical images after 3 months (Fig. 3e-i) show signs of healing

\section{Karger'}




\section{Case Reports in Orthopedic Research}

\begin{tabular}{l|l}
\hline Case Rep Orthop Res 2021;4:255-260 \\
\hline DOI: 10.1159/000521029 & $\begin{array}{l}\text { ○ 2021 The Author(s). Published by S. Karger AG, Basel } \\
\text { www.karger.com/cio }\end{array}$ \\
\hline
\end{tabular}
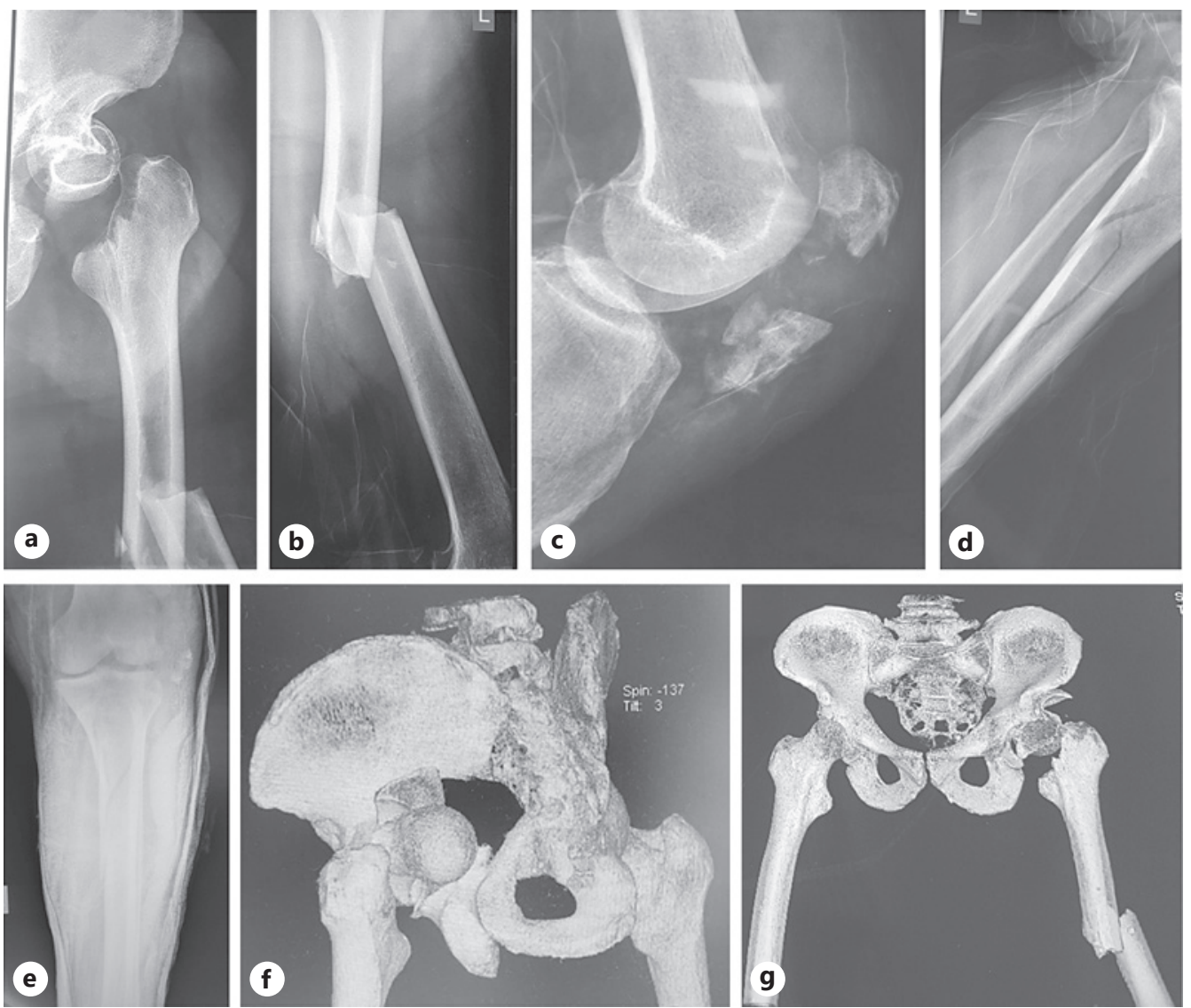

Fig. 1. a-g X-rays show the fracture of the posterior wall of acetabulum, hip dislocation with the femoral neck fracture, and the ipsilateral femoral-shaft fracture. $\mathbf{a}$, $\mathbf{b}$ Lateral view of the knee shows the patella fracture. c Lateral and anteroposterior X-rays show the ipsilateral tibial fracture. $\mathbf{d}, \mathbf{e}$ 3D CT image (f, $\mathbf{g})$.
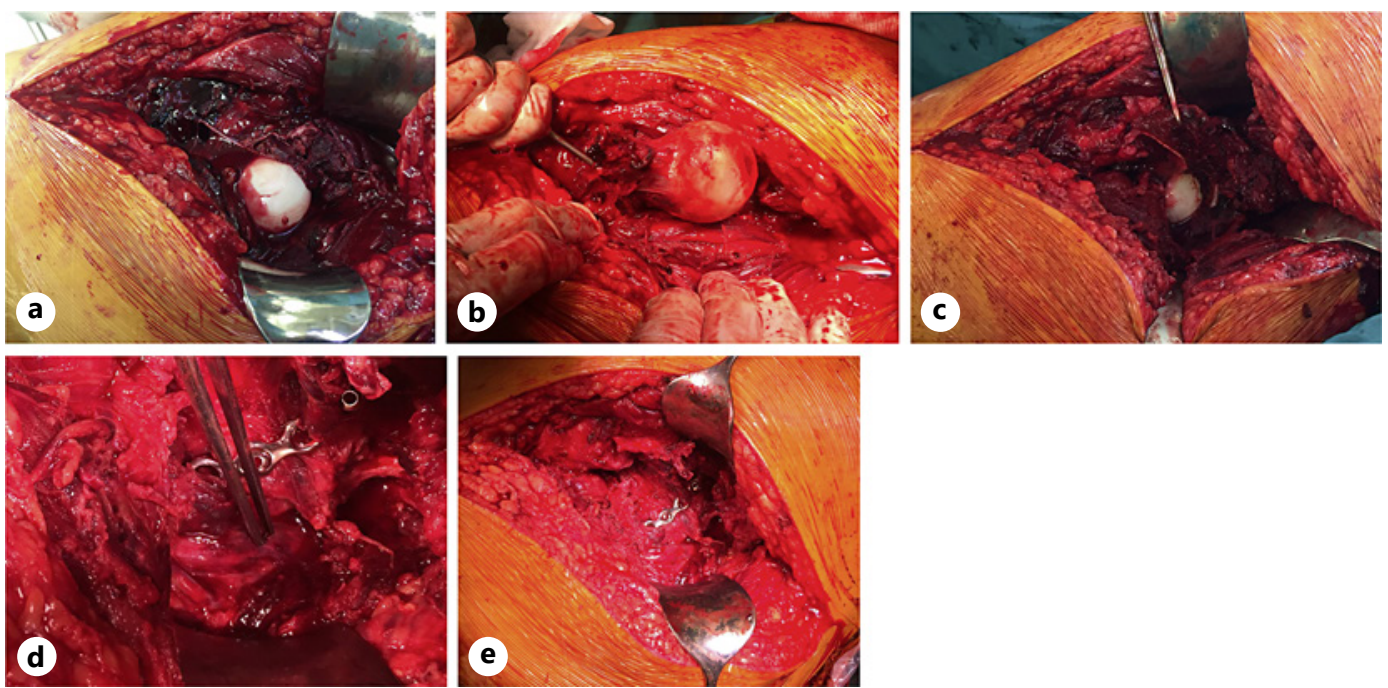

Fig. 2. a-c Intraoperative images show the completely extruded and separated femoral head. a, b Intraoperative appearance after femoral head reduction. c Post-traumatic hematoma on the sciatic nerve. Intraoperative appearance after reduction (d) and fixation of the posterior wall acetabular fracture (e). 


\section{Case Reports in Orthopedic Research}

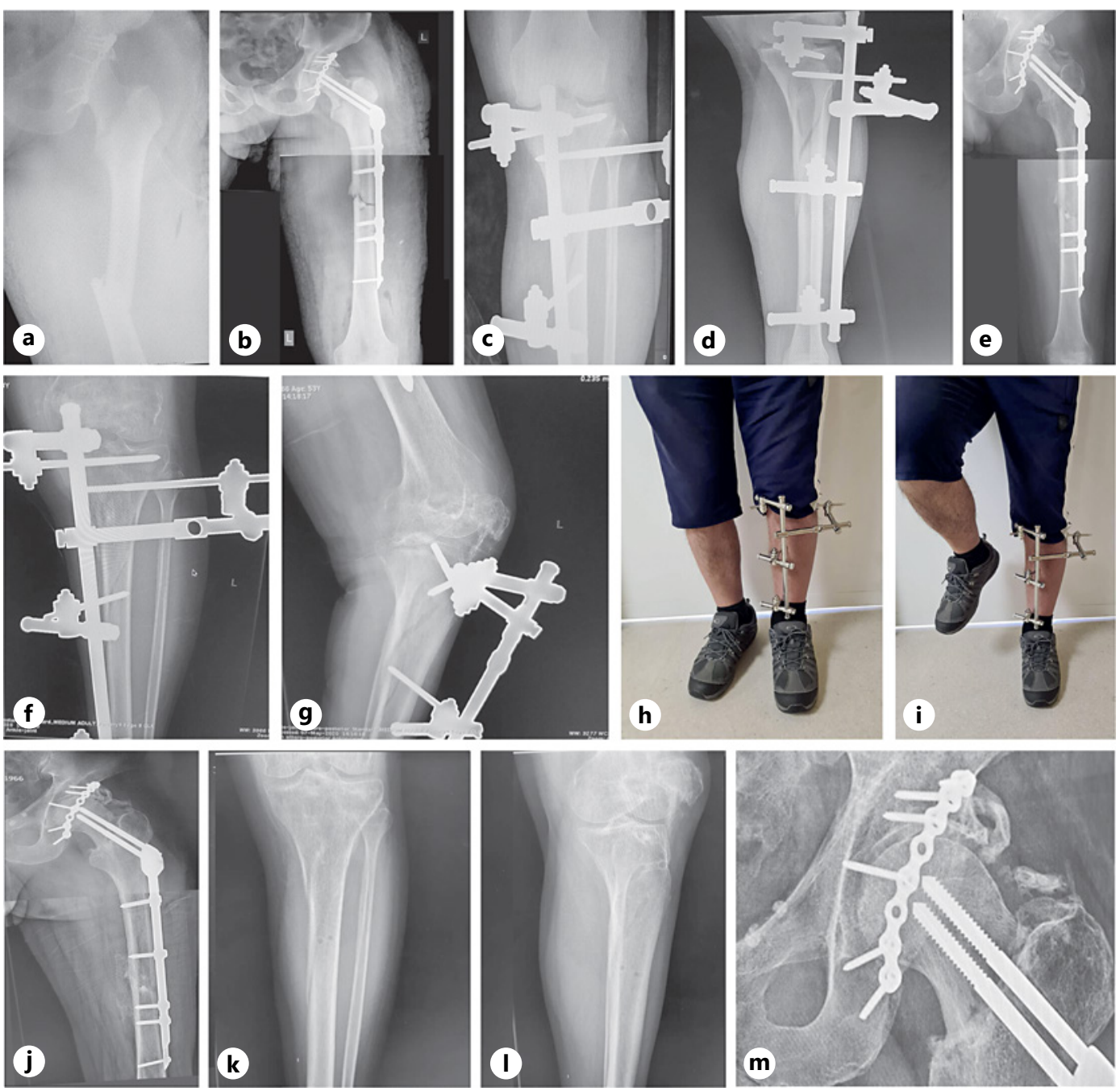

Fig. 3. a-m X-ray after the first surgery. a X-ray after osteosynthesis of the femoral neck and shaft fracture. b X-rays after external fixation of the tibia. $\mathbf{c}, \mathbf{d}$ X-rays 3 months after the injury. $\mathbf{e - g}$ Clinical appearance 3 months after the injury. $\mathbf{h}, \mathbf{i}$ X-rays 14 months after the injury (j-m).

of all fractures, heterotopic ossification was present in the left hip, and the patient walked independently with crutches and full weight-bearing on the left leg. The external fixator was removed from the tibia 6 months after the injury, and the patient continued with physical therapy. After 14 months, the patient walked independently without crutches (online suppl. Video); peroneal nerve is partially recovered; X-rays show complete healing of all fractures; the femoral head is without signs of osteonecrosis; and there are also no signs of secondary osteoarthritis of the hip (Fig. 3j-m). Hip ROM were flexion $100^{\circ}$, abduction $40^{\circ}$, and adduction $15^{\circ}$. Knee flexion was $100^{\circ}$. The PROM score value was 14/20 (70\%) ( $T$-score 44, 9).

\section{Discussion}

Simultaneous ipsilateral injuries of "floating hip" and "floating knee" are very rare and severe, and they occur in high-velocity road traffic accidents. These injuries can be lifethreatening, and many complications can occur during and after treatment [5]. Aggressive 
resuscitation and early surgical treatment within a single surgical intervention or staging surgery represent best approaches when it comes to the treatment of these injuries. Traumatic hip dislocation represents an orthopedic emergency. The time interval from admission to surgery can be out of the utmost importance, and it can affect the final outcome of the treatment. Weekend health care has been associated with poorer outcomes relative to care provided during regular weekday hours. This phenomenon has become known as the "weekend effect" and has been speculated to result from decreased staffing and resource availability, leading to shortfalls in care and poor outcomes [6]. Most of the other delays were due to the need for an appropriately experienced surgeon to be available. Simultaneous ipsilateral "floating-hip" and "floating-knee" injuries represent a great challenge and require extensive surgical experience, careful planning, and teamwork because there are no special protocols for the treatment of serious injuries such as these [1]. The complexity of our injury was severe, and such injuries have shown to be associated with a high chance of osteonecrosis of the femoral head. Unfortunately, severe injuries that we were faced with occurred on the start of the weekend, and the on-duty surgical team was not familiar with the acetabulum surgery. The time interval from injury to surgery in our case was $72 \mathrm{~h}$. Given the severity of the initial hip trauma, osteonecrosis of the femoral head would also occur in the case of immediate reduction since the vascularization of the femoral head was initially destroyed. The organization of the trauma service in the centers that treat these types of injuries must provide the constant presence and availability of experienced surgeons, even on nonworking, weekend days. Osteonecrosis (avascular necrosis) of the femoral head occurs as a consequence of femoral head avascularity. According to Sapkas et al. [7], medial circumflex femoral artery disruption causes osteonecrosis of the femoral head, and the time interval between the injury and the hip reduction has no impact on the development of the osteonecrosis. Other authors recommend urgent reduction of the dislocated hip as this reduces the chances of the development osteonecrosis of the femoral head [8].

\section{Conclusion}

Simultaneous ipsilateral "floating hip" with hip dislocation and femoral neck and shaft fractures associated with "floating-knee" injuries are extremely rare and severe and require wide surgical experience. These injuries can be life-threatening, and many complications can occur during and after treatment. Traumatic hip dislocation with a fracture of the femoral neck required urgent hip reduction, no later than $6 \mathrm{~h}$ after the injury. Every hip reduction after $6 \mathrm{~h}$ from the injury increases the possibility of osteonecrosis of the femoral head. The complexity of our injury was severe, and such injuries have shown to be associated with a high chance of osteonecrosis of the femoral head or secondary osteoarthritis of the hip. Despite the seriousness of the presented injuries, we did not have any complications; 14 months after the injury, the femoral head is still viable, with no signs of femoral head osteonecrosis.

\section{Acknowledgment}

This manuscript is supported by the Medical faculty, University of Nis, Serbia, internal project MFN No. 64, titled "Total hip arthroplasty after earlier acetabular fractures."

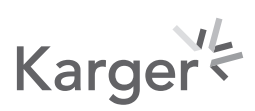


Case Reports

in Orthopedic

Research

\begin{tabular}{l|l}
\hline Case Rep Orthop Res 2021;4:255-260 \\
\hline DOI: 10.1159/000521029 & $\begin{array}{l}\text { @ 2021 The Author(s). Published by S. Karger AG, Basel } \\
\text { www.karger.com/cio }\end{array}$ \\
\hline
\end{tabular}

Milenkovic and Mitkovic: Simultaneous Ipsilateral "Floating-Hip" and "Floating-Knee" Injuries

\section{Statement of Ethics}

Approval and consent of the Ethics Committee of the University Clinical Centre Nis (26349/1 from August 18,2021) have been received for the publication of this article. Written informed consent was obtained from the patient for publication of this case report and any accompanying images and online supplementary video file.

\section{Conflict of Interest Statement}

The authors declare that they have no competing interests.

\section{Funding Sources}

No funds were received in support of this study.

\section{Author Contributions}

S.M. contributed to writing and finalizing the article. M.M. contributed to data collection with editing of the images and radiographs.

\section{Data Availability Statement}

The datasets used and/or analyzed during the current study are available from the corresponding author on reasonable request.

\section{References}

1 Rajasekaran RB, Jayaramaraju D, Palanisami DR, Perumal R, Shanmuganathan R. Ipsilateral acetabular fracture with displaced femoral head and femoral shaft fracture: a complex floating hip injury. Case Rep Orthop. 2018;2018:4937472.

2 Yashavantha Kumar C, Nalini KB, Nagaraj P, Jawali A. Ipsilateral floating hip and floating knee: a rare entity. J Orthop Case Rep. 2013;3(3):3-6.

3 Anand V, Deviprasad Praba K, Babar S, Babu M. Ipsilateral floating hip and knee, or floating lower limb: a rare case report. Trauma Case Reports. 2021;31:100390.

4 Benabbouha A, Boussouga M, Fjouji S, Lamkhanter A, Jaafar A. Simultaneous ipsilateral floating hip and knee: a complex combination and difficult surgical challenge. Case Reports in Orthopedics. 2020;2020:1.

5 Cannada LK, Justin MH, Preston JB, Heidi I, Hassan M, Jason H, et al. Treatment and complications of patients with ipsilateral acetabular and femur fractures. J Orthop Trauma. 2017;31(12):650-6.

6 Zapf MA, Kothari AN, Markossian T, Gupta GN, Blackwell RH, Wai PY, et al. The "weekend effect" in urgent general operative procedures. Surgery. 2015;158(2):508-14.

7 Sapkas G, Pantazopoulos T, Karanikas E, Hartofilakidis-Garofalidis G. Effect of hip dislocation on the blood supply to the femoral head. An experimental study in rabbits. Acta Orthop Scand. 1983;54(2):204-9.

8 Hougaard K, Thomsen PB. Traumatic posterior dislocation of the hip- prognostic factors influencing the incidence of avascular necrosis of the femoral head. Arch Orthop Trauma Surg. 1986;106:32-5. 